\title{
Clinical implications of anti-thyroglobulin antibody measurement before surgery in thyroid cancer
}

\author{
Kwanhoon Jo ${ }^{1}$ and Dong-Jun Lim ${ }^{2}$
}

\author{
${ }^{1}$ Division of Endocrinology and \\ Metabolism, Department of Internal \\ Medicine, College of Medicine, \\ Incheon St. Mary's Hospital, The \\ Catholic University of Korea, \\ Incheon; ${ }^{2}$ Division of Endocrinology \\ and Metabolism, Department \\ of Internal Medicine, College of \\ Medicine, Seoul St. Mary's Hospital, \\ The Catholic University of Korea, \\ Seoul, Korea
}

Received: August 4, 2018

Accepted: October 5, 2018

\section{Correspondence to}

Dong-Jun Lim, M.D.

Division of Endocrinology and Metabolism, Department of Internal Medicine, College of Medicine, Seoul St. Mary's Hospital,

The Catholic University of Korea, 222 Banpo-daero, Seocho-gu,

Seoul o6591, Korea

Tel: +82-2-2258-6009

Fax: +82-2-599-3589

E-mail:1dj6026@catholic.ac.kr
Thyroglobulin antibody $(\mathrm{TgAb})$ is a class $\mathrm{G}$ immunoglobulin and a conventional marker for thyroid autoimmunity. From a clinical perspective, TgAb is less useful than thyroid peroxidase antibodies for predicting thyroid dysfunction. However, $\mathrm{TgAb}$ is found more frequently in differentiated thyroid cancer (DTC) and can interfere with thyroglobulin ( $\mathrm{Tg}$ ) measurements, which are used to monitor the recurrence or persistence of DTC. Recent studies suggested a small but consistent role for preoperative $\mathrm{TgAb}$ in predicting DTC in thyroid nodules, and in reflecting adverse tumor characteristics or prognosis, including lymph node metastasis, but this is still controversial. Postoperative $\mathrm{TgAb}$ can serve as a biomarker for remnant thyroid tissue, so follow-up measures of $\mathrm{TgAb}$ are useful for predicting cancer recurrence in DTC patients. Since high serum $\mathrm{TgAb}$ levels may also affect the fine needle aspiration washout $\mathrm{Tg}$ levels from suspicious lymph nodes of DTC patients, it is important to use caution when interpreting the washout $\mathrm{Tg}$ levels in patients who are positive for $\mathrm{TgAb}$.

Keywords: Anti-thyroglobulin; Thyroid neoplasms

\section{INTRODUCTION}

Differentiated thyroid cancer (DTC) is the most common endocrine cancer, and its incidence has been increasing worldwide. DTC is found in $7 \%$ to $15 \%$ of thyroid nodules, and it has a good prognosis [1]. The most common type of DTC is papillary thyroid cancer (PTC), comprising more than $90 \%$ of all thyroid cancers, whereas follicular thyroid cancer is less common, comprising $7 \%$ to $8 \%$ of thyroid cancers in Korea $[2,3]$.
Lobectomy or total thyroidectomy is generally the treatment of choice, except for some microcarcinomas. After thyroidectomy, thyroid hormone replacement and radioactive iodine (RI) for remnant ablation are administered [4].

Serum thyroglobulin $(\mathrm{Tg})$ is the most sensitive established biomarker of DTC after total thyroidectomy and RI. Tg is produced only in normal thyroid tissue or in thyroid cancer tissue, so measuring $\mathrm{Tg}$ levels in the blood is useful for the early detection of recurrent or 
residual disease in DTC patients who have undergone total thyroidectomy and RI. Disease-free individuals have extremely low Tg levels.

Tg levels could be affected by the presence of thyroglobulin antibody (TgAb), which falsely lowers or elevates serum $\mathrm{Tg}$ levels $[5,6]$. Thus, current cancer management guidelines mandate that $\mathrm{Tg}$ testing should always include the measurement of TgAb. Generally, an elevated $\mathrm{TgAb}$ level alone rarely indicates any specific disease states or changes, except during the post-operative follow-up of DTC patients with positive TgAb levels. However, studies have shown that the clinical use of $\mathrm{TgAb}$ has the potential to improve patient care. In this article, we summarize the ways that $\mathrm{TgAb}$ is being used in a variety of clinical settings in the thyroid cancer field.

\section{CHARACTERISTICS OF TgAb}

There are many thyroid-related antibodies that target specific proteins within or around the thyroid follicular cells, including thyroid peroxidase (TPO), thyroid-stimulating hormone (TSH) receptor, $\mathrm{Tg}$, and, rarely, carbonic anhydrase 2, megalin, triiodothyronine, thyroxine, sodium iodide symporter (NIS), and pendrin [7]. Thyroid-related antibodies are usually related to thyroid autoimmune diseases: TPO antibodies (TPOAb) and TgAb indicate Hashimoto's thyroiditis (HT), and TSH receptor antibodies indicate Graves' disease (GD).

$\mathrm{TgAb}$ was the first antibody found in patients with autoimmune thyroid disease (AITD) as described by Doniach and Roitt in 1956 [8,9]. Most TgAbs are classified as immunoglobulin $\mathrm{G}(\mathrm{IgG})$, though some are IgA. It is an intra-follicular antibody that can bind to immune cells and antigens with or without tissue destruction. Massive destruction of the thyroid gland induces structural changes in $\mathrm{Tg}$, leading to antibody production against Tg. Major T-cell epitopes on Tg require iodination for recognition by autoreactive T-cells [10]. This explains the higher incidence of positive $\mathrm{TgAb}$ levels in patients with an excessive iodine intake [11]. However, high Tg levels in blood do not necessarily induce antibody production. The TgAb level depends on antigen exposure time [7]. There are nearly $40 \mathrm{Tg}$ epitopes, of which only a few are immunogenic [12].
TgAb itself does not cause thyroid cell destruction, and it does not fix complement because the epitopes are too widely spaced to allow cross-linking [13].

\section{TgAb AND DTC}

Studies have suggested that $\operatorname{TgAb}$ plays a role in the relationship between autoimmune thyroiditis and thyroid cancer; most of these studies have been pathological studies, though some are serological studies [14-16]. However, it is unclear what roles the thyroid autoantibodies that indicate thyroiditis play in the pathogenesis of thyroid cancer [7].

The prevalence of $\mathrm{TgAb}$ is approximately 1.5-fold higher in patients with DTC than in the general population with benign nodules (30.8\% vs. 19.6\%) [17]. TgAb is found more frequently in patients with PTC than in patients with follicular carcinomas [18]. Interestingly, the patterns of $\mathrm{TgAb}$ differ between patients with AITD and non-AITD patients, including those with DTC. Latrofa et al. [12] used human recombinant TgAb-Fab to investigate $\mathrm{TgAb}$ recognition in sera from patients with non-AITD thyroid diseases (nontoxic multinodular goiter and PTC) and AITD (HT and GD). They found that region $\mathrm{A}$ is the major immunodominant region of $\mathrm{Tg}$, because the highest levels of inhibition were induced by TgAb-Fab of region A in all patients (> 50\%) regardless of AITD. The inhibition levels were significantly higher in AITD patients than in non-AITD patients [12].

\section{PREOPERATIVE TgAb AS A PREDICTOR OF THYROID CANCER}

Positive serum $\mathrm{TgAb}$ is more than twice as prevalent in DTC patients than in the general population $(25 \% \mathrm{vs}$. 10\%) [14]. While TPOAb are believed to fix complement and can be used to diagnose HT and hypothyroidism more accurately than $\mathrm{TgAb}, \mathrm{TgAb}$ may be more tumor-specific than TPOAb [19]. In particular, high TgAb levels can weakly predict thyroid carcinomas in patients with thyroid nodules, unlike TPOAb [17].

One study retrospectively evaluated a total of 2088 patients with thyroid nodules who received fine needle aspiration (FNA), and found that thyroid cancer was sig- 
nificantly associated with positive $\mathrm{TgAb}$ (odds ratio [OR], 1.61) [17]. A similar result was obtained by Vasileiadis et al. [20], who found that in patients who received a total thyroidectomy for any reason, preoperative measurements found $\mathrm{TgAb}$ more frequently in patients with malignant disease than in those with benign disease (38.2\% vs. 19.8\%). In another study of 2,562 patients who underwent FNA cytology (FNAC), suspicious cytology was detected more frequently in $\mathrm{TgAb}$-positive patients than in those without $\mathrm{TgAb}$ (9.4\% vs 5.7\%), and isolated $\mathrm{TgAb}$ was a mild risk factor for thyroid cancer (OR, 1.74) [21]. Hosseini et al. [22] grouped patients according to $\mathrm{TgAb}$ level and found out that the prevalence of malignancy was higher in the positive TgAb group (65.38\% vs. 50.42\%). Qin el al. [23] also grouped patients according to TgAb level (o, 4, 11, 40, 100, and $500 \mathrm{IU} / \mathrm{mL}$ ), and found that the prevalence of DTC was significantly higher in the high $\mathrm{TgAb}$ groups ( $\geq 100 \mathrm{IU} / \mathrm{mL}$ ) than in the low $\mathrm{TgAb}$ groups (<100 IU/mL).

However, when thyroid autoantibodies were considered as a marker for AITD, there was no significant difference in thyroid malignancy rates between AITD and non-AITD groups. Anil et al. [24] recruited patients with newly diagnosed thyroid nodules and evaluated thyroid autoantibodies before FNA. The FNA results revealed no differences in malignancy rates between the non-AITD and AITD groups. However, AITD patients were positive for at least one thyroid autoantibody, so this study could not detect the effects of TgAb alone on malignancy rates. Rago et al. [25] found similar results, but they also did not distinguish between $\mathrm{TgAb}$ and TPOAb.

Overall, these results suggest that unlike TPOAb, $\mathrm{TgAb}$ could be a weak but confident predictive marker for PTC in patients presenting with thyroid nodules (Table 1) [17,20-23,26-30]. TgAb may detect newly developed epitopes of $\mathrm{Tg}$ derived from thyroid cancer cells. Tg from benign follicular cells may be unlikely to develop any novel cancer-specific epitopes. The predictive value of $\mathrm{TgAb}$ for thyroid cancer before FNA is not very powerful and is controversial; therefore, other clinical parameters should be combined with TgAb to increase the diagnostic power of TgAb.

\section{PREOPERATIVE TgAb LEVELS IN PREDICTING THE PROGNOSIS OF THYROID CANCER}

It is generally accepted that thyroid cancer with AITD has a more favorable prognosis compared to thyroid cancer without AITD [14,16,31]. Most studies, however, have not examined the effects of TPOAb or TgAb separately, but rather the combined effects of both thyroid autoantibodies or the entire AITD pathology. Therefore, studies evaluating the effects of $\mathrm{TgAb}$ individually were needed to understand the role of $\mathrm{TgAb}$ in cancer prognosis.

Several studies were conducted on the prognostic effects of $\mathrm{TgAb}$ on thyroid cancer in perioperative settings (Table 2). Durante et al. [32] showed that there were more cases of persistent/recurrent disease in thyroid cancer patients with $\mathrm{TgAb}$ than in those without $\mathrm{TgAb}$, although these data were not preoperative; instead, $\mathrm{TgAb}$ values were obtained 1 to 12 months after thyroidectomy. On the other hand, a study based on a nationwide multicenter registry of thyroid cancer in the United States showed no significant association of $\mathrm{TgAb}$ with the stage of disease, disease-free survival, or overall survival [33]. Although these studies found contradictory results, it is interesting to see the potential role of $\mathrm{TgAb}$ in the prognosis of thyroid cancer and in its underlying mechanisms.

Since then, there have been several studies reporting a correlation between $\mathrm{TgAb}$ and lymph node metastasis in DTC. Vasileiadis et al. [20] reported that DTC patients with $\mathrm{TgAb}$ showed more frequent lymph node metastasis than those without $\operatorname{TgAb}$ (20.3\% vs. 10.0\%). Another study also found that elevated preoperative serum TgAb levels in DTC patients were significantly associated with adverse primary tumor characteristics, including nonencapsulated tumors, lymphatic invasion, and lymph node metastasis [34]. Similarly, Zhang et al. [35] found that DTC patients with TgAb had a significantly higher rate of metastasis of cervical lymph nodes, and that lymph node stage was the only independent indicator for persistent $\mathrm{TgAb}$ (OR, 3.183). Qin et al. [23] also reported that patients with TgAb showed a higher rate of extrathyroid invasion (30.4\% vs. 17.1\%, p $=0.031$ ).

One small study by Li et al. [36] evaluated the protein expression of specific invasion-related genes (BRAF 

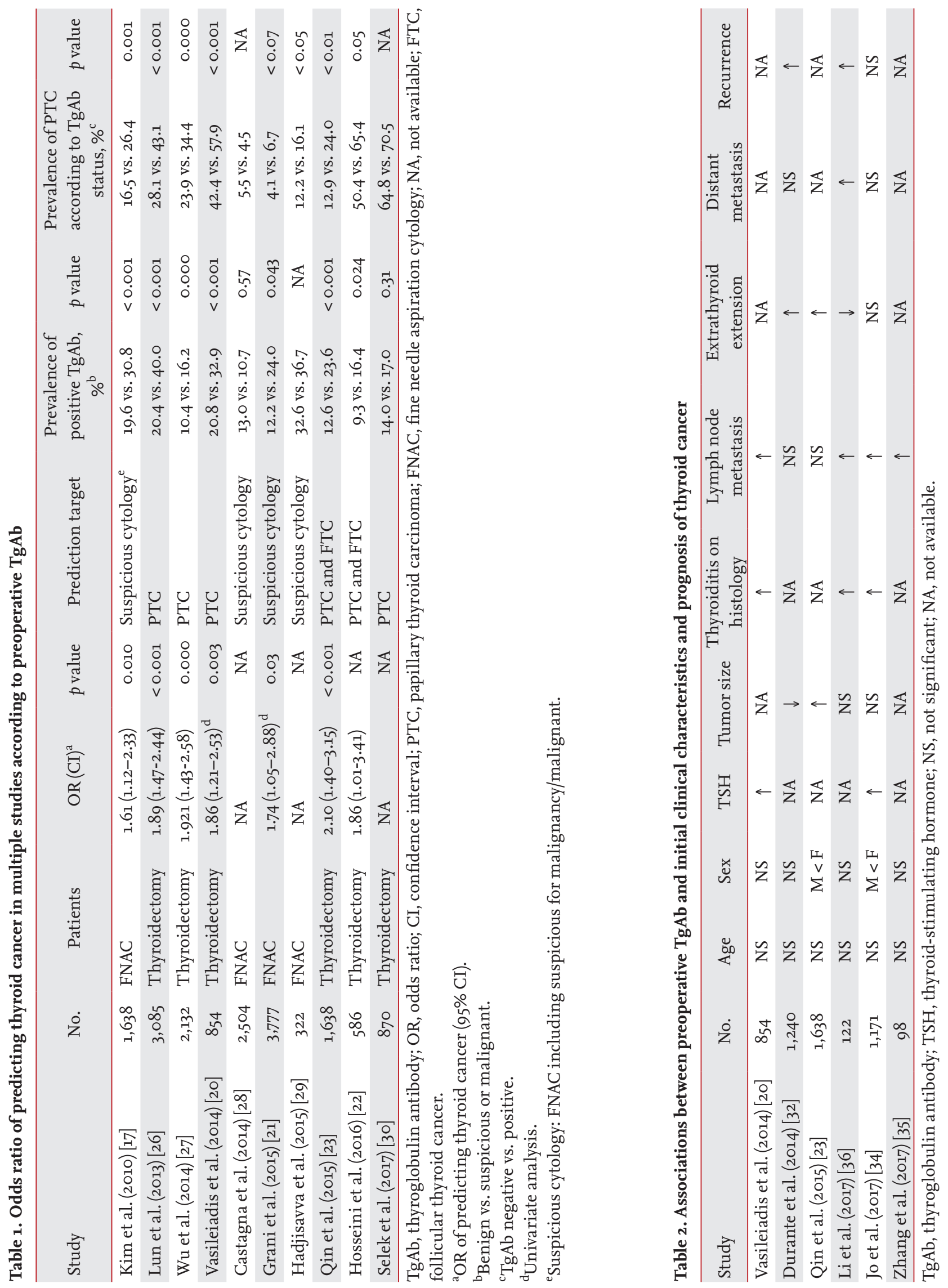
$\mathrm{V} 600 \mathrm{E}$ and nuclear factor $\mathrm{\kappa B}[\mathrm{NF}-\mathrm{\kappa B}])$ in thyroid cancer tissues to test whether TgAb is associated with the expression of these genes. The protein expression levels of BRAFV6ooE and NF- $\kappa$ B were greater in TgAb-positive patients compared to TgAb-negative patients, and a multivariate analysis found that $\mathrm{TgAb}$ was an independent factor for predicting lymph node metastasis in thyroid cancer. However, the small number of subjects and the lack of a mechanism for the observed patterns make this result difficult to interpret.

If metastasis or invasion develops from a primary tumor, cancer cells are confined within the site and will induce a cancer-specific immune response, which may in turn cause TgAb levels to rise [37,38]. One recent study used genomic profiling of thyroid cancer to reveal that somatic mutations of $\mathrm{Tg}$ derived from metastatic lesions might play a significant role in the pathogenesis of PTC and development of metastasis [39]. Newly developed Tg mutations in this study were associated with a poor prognosis, and we presume these new Tg mutations would induce TgAb. Unfortunately, $\mathrm{TgAb}$ was not measured in this study.

\section{POSTOPERATIVE TgAb FOLLOW-UP}

High TgAb levels could cause Tg levels to be undetectable. Because of this, follow-up measurements of TgAb levels are useful for detecting persistent or recurrent disease after total thyroidectomy and RI remnant ablation of DTC [4]. Therefore, rising TgAb levels after initial treatment are evaluated as a 'biochemical incomplete response,' and these patients should be carefully monitored to detect recurrent or persistent disease [4].

$\mathrm{TgAb}$ levels may transiently rise postoperatively as an apparent immune reaction to released tissue particles after surgery, and they may rise after RI [40]. After total thyroidectomy and RI, all antibodies disappeared progressively, with a median disappearance time for $\mathrm{TgAb}$ of about 3 years $[40,41]$. Although the pathophysiologic importance of $\mathrm{TgAb}$ after total thyroidectomy is unclear, the persistence of $\mathrm{TgAb}$ or increasing titers more than 1 year after thyroidectomy and RI probably indicates the presence of residual thyroid tissue, and possibly an increased risk of recurrence [14]. Furthermore, sequential changes in TgAb titers are a reliable predic- tor of disease prognosis and therefore serve as a useful factor for clinical decision-making [42]. In a study of thyroid cancer patients with undetectable serum $\mathrm{Tg}$ concentrations, $18 \%$ of patients with serum $\mathrm{TgAb}$ concentrations $>100 \mathrm{U} / \mathrm{mL}$ had a recurrence, compared with only $1 \%$ of patients with serum $\mathrm{TgAb} \leq 100 \mathrm{U} / \mathrm{mL}$ [43].

We previously reported that in DTC patients who previously had total thyroidectomy and high-dose RI remnant ablation, thyroid bed RI uptake at the postoperative 6- to 12-month whole-body scan showed a close association with higher $\mathrm{TgAb}$ levels, compared to no thyroid bed uptake [44]. Dewi et al. [45] also reported that higher post-RI TgAb levels were significantly correlated with unsuccessful RI therapy (OR, 5.379; $p=$ 0.007), which indicates that $\mathrm{TgAb}$ could serve as a surrogate marker for successful RI. We assume that the existence of $\mathrm{TgAb}$ represents remnant thyroid tissue, because $\mathrm{TgAb}$ can be detected as a response to remaining Tg.

A study by Trimboli et al. [46] evaluated the prognostic impact of pre-RI TgAb levels on long-term outcomes for TgAb-positive DTC patients. Positive TgAb levels before RI increased the risk of disease recurrence over time (OR, 3.57), and disease-free survival was higher in TgAb-negative patients (hazard ratio, 2.59).

\section{FNA WASHOUT TG AND SERUM TgAb LEVELS}

FNAC of suspicious lymph nodes in DTC patients frequently shows false-negative results due to easily missed cancer cells in cases with an insufficient number of metastatic cancer cells within the lymph nodes. Therefore, measuring Tg levels in FNA washout (FNA$\mathrm{Tg}$ ), in addition to FNAC, has been the best supplementary diagnostic tool to increase the diagnostic accuracy of FNAC [47]. Increased serum TgAb levels could lower serum $\mathrm{Tg}$ concentrations, so measurements of $\mathrm{Tg}$ should always be accompanied by TgAb testing, as indicated by clinical management guidelines [4]. This leads to the question of whether increased serum TgAb levels could interfere with FNA-Tg levels in metastatic lymph nodes of DTC patients.

Several studies found that the presence of serum TgAb could lower FNA-Tg levels, and therefore mea- 
suring serum $\mathrm{TgAb}$ levels may be helpful in DTC patients who are bound to FNAC with FNA-Tg on suspicious lymph nodes [48-51]. One study demonstrated that when applying the same FNA-Tg cutoff used in the $\mathrm{TgAb}$-negative group to the $\mathrm{TgAb}$-positive group to detect metastatic lymph nodes in DTC patients, the sensitivity and positive predictive value of FNA-Tg were reduced by more than $10 \%$ [51]. A similar result was obtained in a postoperative setting: the TgAb-positive group showed significantly lower FNA-Tg levels than the $\mathrm{TgAb}$-negative group in metastatic lymph nodes, and thus showed lower sensitivity and negative predictive values with the same FNA-Tg levels [49].

In multiple studies, however, high serum TgAb levels showed no interference with FNA-Tg sensitivity [52,53]. Duval et al. [54] reported that the measurement of FNA$\mathrm{Tg}$ is useful for evaluating suspicious lymph nodes in patients with DTC, regardless of TgAb. Boi et al. [53] measured TgAb levels in FNA washout fluid in a limited number of patients, and found that $\mathrm{TgAb}$ was undetectable in patients with negative serum $\mathrm{TgAb}$, but a small number of patients with positive serum $\mathrm{TgAb}$ (two of eight) showed positive $\mathrm{TgAb}$ in washout fluid. FNATg levels from metastatic lymph nodes were lower than those found in TgAb-negative FNA washout fluids, but this difference might not be significant in the diagnostic process. One explanation for why FNA-Tg levels are much higher than TgAb levels in FNA washout fluid is that it may overcome interference by $\mathrm{TgAb}$, due to high Tg levels in metastatic lymph nodes leading to saturated TgAb-binding sites. Thus, the measurement of serum $\mathrm{TgAb}$ at the time of FNA-Tg is not recommended in current clinical management guidelines [4]. Additional studies are needed to confirm the interfering effects of serum $\mathrm{TgAb}$ levels on FNA washout $\mathrm{Tg}$ levels.

\section{CONCLUSIONS}

It remains challenging to interpret the diagnostic and prognostic implications of $\mathrm{TgAb}$ levels before surgery for DTC patients with TgAb, although basic and clinical researchers have uncovered secret messages from this antibody over the last two decades. Future research should focus on understanding the epitope-specific immunological responses of $\mathrm{TgAb}$ in thyroid cancer, as several studies have suggested unique immunological roles for $\mathrm{TgAb}$.

\section{Conflict of interest}

No potential conflict of interest relevant to this article was reported.

\section{REFERENCES}

1. Hegedus L. Clinical practice: the thyroid nodule. N Engl J Med 2004;351:1764-1771.

2. Hori M, Katanoda K. Morphological distribution of thyroid cancer from Cancer Incidence in Five Continents Vol. X. Jpn J Clin Oncol 2015;45:1182.

3. Cho BY, Choi HS, Park YJ, et al. Changes in the clinicopathological characteristics and outcomes of thyroid cancer in Korea over the past four decades. Thyroid 2013;23:797-804.

4. Haugen BR, Alexander EK, Bible KC, et al. 2015 American Thyroid Association management guidelines for adult patients with thyroid nodules and differentiated thyroid cancer: the American Thyroid Association Guidelines task force on thyroid nodules and differentiated thyroid cancer. Thyroid 2016;26:1-133.

5. Bayer MF, Kriss JP. Immunoradiometric assay for serum thyroglobulin: semiquantitative measurement of thyroglobulin in antithyroglobulin-positive sera. J Clin Endocrinol Metab 1979;49:557-564.

6. Schneider AB, Pervos R. Radioimmunoassay of human thyroglobulin: effect of antithyroglobulin autoantibodies. J Clin Endocrinol Metab 1978;47:126-137.

7. Fröhlich E, Wahl R. Thyroid autoimmunity: role of anti-thyroid antibodies in thyroid and extra-thyroidal diseases. Front Immunol 2017;8:521.

8. Roitt IM, Doniach D, Campbell PN, Hudson RV. Auto-antibodies in Hashimoto's disease (lymphadenoid goitre). Lancet 1956;271:820-821.

9. Rose NR, Witebsky E. Studies on organ specificity. V. Changes in the thyroid glands of rabbits following active immunization with rabbit thyroid extracts. J Immunol 1956;76:417-427.

10. Barin JG, Talor MV, Sharma RB, Rose NR, Burek CL. Iodination of murine thyroglobulin enhances autoimmune reactivity in the NOD.H2 mouse. Clin Exp Immunol 2005;142:251-259.

11. Li Y, Teng D, Shan Z, et al. Antithyroperoxidase and anti- 
thyroglobulin antibodies in a five-year follow-up survey of populations with different iodine intakes. J Clin Endocrinol Metab 2008;93:1751-1757.

12. Latrofa F, Ricci D, Grasso L, et al. Characterization of thyroglobulin epitopes in patients with autoimmune and non-autoimmune thyroid diseases using recombinant human monoclonal thyroglobulin autoantibodies. J Clin Endocrinol Metab 2008;93:591-596.

13. Caturegli P, Kuppers RC, Mariotti S, et al. IgG subclass distribution of thyroglobulin antibodies in patients with thyroid disease. Clin Exp Immunol 1994;98:464-469.

14. Spencer CA, Takeuchi M, Kazarosyan M, et al. Serum thyroglobulin autoantibodies: prevalence, influence on serum thyroglobulin measurement, and prognostic significance in patients with differentiated thyroid carcinoma. J Clin Endocrinol Metab 1998;83:1121-1127.

15. Feldt-Rasmussen U, Rasmussen AK. Autoimmunity in differentiated thyroid cancer: significance and related clinical problems. Hormones (Athens) 2010;9:109-117.

16. Kebebew E, Treseler PA, Ituarte PH, Clark OH. Coexisting chronic lymphocytic thyroiditis and papillary thyroid cancer revisited. World J Surg 2001;25:632-637.

17. Kim ES, Lim DJ, Baek KH, et al. Thyroglobulin antibody is associated with increased cancer risk in thyroid nodules. Thyroid 2010;20:885-891.

18. Braverman LE, David DS, Werner SC, Ingbar SH. Werner \& Ingbar's the Thyroid: A Fundamental and Clinical Text. Philadelphia (PA): Lippincott Williams \& Wilkins, 2013.

19. McLachlan SM, Rapoport B. Why measure thyroglobulin autoantibodies rather than thyroid peroxidase autoantibodies? Thyroid 2004;14:510-520.

20. Vasileiadis I, Boutzios G, Charitoudis G, Koukoulioti E, Karatzas T. Thyroglobulin antibodies could be a potential predictive marker for papillary thyroid carcinoma. Ann Surg Oncol 2014;21:2725-2732.

21. Grani G, Calvanese A, Carbotta G, et al. Thyroid autoimmunity and risk of malignancy in thyroid nodules submitted to fine-needle aspiration cytology. Head Neck 2015;37:260-264.

22. Hosseini S, Payne RJ, Zawawi F, et al. Can preoperative thyroglobulin antibody levels be used as a marker for well differentiated thyroid cancer? J Otolaryngol Head Neck Surg 2016;45:31.

23. Qin J, Yu Z, Guan H, Shi L, et al. High thyroglobulin antibody levels increase the risk of differentiated thyroid carcinoma. Dis Markers 2015;2015:648670.
24. Anil C, Goksel S, Gursoy A. Hashimoto's thyroiditis is not associated with increased risk of thyroid cancer in patients with thyroid nodules: a single-center prospective study. Thyroid 2010;20:601-606.

25. Rago T, Di Coscio G, Ugolini C, et al. Clinical features of thyroid autoimmunity are associated with thyroiditis on histology and are not predictive of malignancy in 570 patients with indeterminate nodules on cytology who had a thyroidectomy. Clin Endocrinol (Oxf) 2007;67:363-369.

26. Lun $\mathrm{Y}, \mathrm{Wu} X, \mathrm{Xia} Q$, et al. Hashimoto's thyroiditis as a risk factor of papillary thyroid cancer may improve cancer prognosis. Otolaryngol Head Neck Surg 2013;148:396-402.

27. Wu X, Lun $\mathrm{Y}$, Jiang $\mathrm{H}$, et al. Coexistence of thyroglobulin antibodies and thyroid peroxidase antibodies correlates with elevated thyroid-stimulating hormone level and advanced tumor stage of papillary thyroid cancer. Endocrine 2014;46:554-560.

28. Castagna MG, Belardini V, Memmo S, et al. Nodules in autoimmune thyroiditis are associated with increased risk of thyroid cancer in surgical series but not in cytological series: evidence for selection bias. J Clin Endocrinol Metab 2014;99:3193-3198.

29. Hadjisavva IS, Dina R, Talias MA, Economides PA. Prevalence of cancer in patients with thyroid nodules in the island of cyprus: predictive value of ultrasound features and thyroid autoimmune status. Eur Thyroid J 2015;4:123128.

30. Selek A, Cetinarslan B, Tarkun I, Canturk Z, Ustuner B, Akyay Z. Thyroid autoimmunity: is really associated with papillary thyroid carcinoma? Eur Arch Otorhinolaryngol 2017;274:1677-1681.

31. Kim EY, Kim WG, Kim WB, et al. Coexistence of chronic lymphocytic thyroiditis is associated with lower recurrence rates in patients with papillary thyroid carcinoma. Clin Endocrinol (Oxf) 2009;71:581-586.

32. Durante C, Tognini S, Montesano T, et al. Clinical aggressiveness and long-term outcome in patients with papillary thyroid cancer and circulating anti-thyroglobulin autoantibodies. Thyroid 2014;24:1139-1145.

33. McLeod DS, Cooper DS, Ladenson PW, et al. Prognosis of differentiated thyroid cancer in relation to serum thyrotropin and thyroglobulin antibody status at time of diagnosis. Thyroid 2014;24:35-42.

34. Jo K, Kim MH, Ha J, et al. Prognostic value of preoperative anti-thyroglobulin antibody in differentiated thyroid cancer. Clin Endocrinol (Oxf) 2017;87:292-299. 
35. Zhang T, Song LP, Zhao T, Gao W, Lin YS. Effect of lymph node metastasis on change of positive thyroglobulin antibody in differentiated thyroid carcinoma after initial treatment. Zhongguo Yi Xue Ke Xue Yuan Xue Bao 2017;39:539-543.

36. Li W, Ming H, Sun D, et al. The relationship between BRAFV6ooE, NF- $\mathrm{B}$ and TgAb expression in papillary thyroid carcinoma. Pathol Res Pract 2017;213:183-188.

37. Nishikawa M, Otsuki T, Ota A, et al. Induction of tumorspecific immune response by gene transfer of Hsp7ocell-penetrating peptide fusion protein to tumors in mice. Mol Ther 2010;18:421-8.

38. Boon T, Gajewski TF, Coulie PG. From defined human tumor antigens to effective immunization? Immunol Today 1995;16:334-6.

39. Siraj AK, Masoodi T, Bu R, et al. Genomic profiling of thyroid cancer reveals a role for thyroglobulin in metastasis. Am J Hum Genet 2016;98:1170-1180.

40. Gorges R, Maniecki M, Jentzen W, et al. Development and clinical impact of thyroglobulin antibodies in patients with differentiated thyroid carcinoma during the first 3 years after thyroidectomy. Eur J Endocrinol 2005;153:4955 .

41. Chiovato L, Latrofa F, Braverman LE, et al. Disappearance of humoral thyroid autoimmunity after complete removal of thyroid antigens. Ann Intern Med 2003;139(5 Pt 1):346-351.

42. Hsieh CJ, Wang PW. Sequential changes of serum antithyroglobulin antibody levels are a good predictor of disease activity in thyroglobulin-negative patients with papillary thyroid carcinoma. Thyroid 2014;24:488-493.

43. Kim WG, Yoon JH, Kim WB, et al. Change of serum antithyroglobulin antibody levels is useful for prediction of clinical recurrence in thyroglobulin-negative patients with differentiated thyroid carcinoma. J Clin Endocrinol Metab 2008;93:4683-4689.

44. Lim DJ, O JH, Kim MH, et al. Clinical significance of observation without repeated radioiodine therapy in differentiated thyroid carcinoma patients with positive surveillance whole-body scans and negative thyroglobulin. Korean J Intern Med 2010;25:408-414.

45. Dewi AR, Darmawan B, Kartamihadja AH, Hidayat B, Masjhur JS. Antithyroglobulin antibody as a marker of successful ablation therapy in differentiated thyroid cancer.
World J Nucl Med 2017;16:15-20.

46. Trimboli P, Zilioli V, Imperiali M, Giovanella L. Thyroglobulin autoantibodies before radioiodine ablation predict differentiated thyroid cancer outcome. Clin Chem Lab Med 2017;55:1995-2001.

47. Snozek CL, Chambers EP, Reading CC, et al. Serum thyroglobulin, high-resolution ultrasound, and lymph node thyroglobulin in diagnosis of differentiated thyroid carcinoma nodal metastases. J Clin Endocrinol Metab 2007;92:4278-81.

48. Shin HJ, Lee HS, Kim EK, Moon HJ, Lee JH, Kwak JY. A study on serum antithyroglobulin antibodies interference in thyroglobulin measurement in fine-needle aspiration for diagnosing lymph node metastasis in postoperative patients. PLoS One 2015;10:e0131096.

49. Jeon MJ, Park JW, Han JM, et al. Serum antithyroglobulin antibodies interfere with thyroglobulin detection in fine-needle aspirates of metastatic neck nodes in papillary thyroid carcinoma. J Clin Endocrinol Metab 2013;98: 153-160.

50. Fiore E, Giustarini E, Mammoli C, et al. Favorable predictive value of thyroid autoimmunity in high aggressive breast cancer. J Endocrinol Invest 2007;30:734-738.

51. Jo K, Kim MH, Lim Y, et al. Lowered cutoff of lymph node fine-needle aspiration thyroglobulin in thyroid cancer patients with serum anti-thyroglobulin antibody. Eur J Endocrinol 2015;173:489-497.

52. Baskin HJ. Detection of recurrent papillary thyroid carcinoma by thyroglobulin assessment in the needle washout after fine-needle aspiration of suspicious lymph nodes. Thyroid 2004;14:959-963.

53. Boi F, Baghino G, Atzeni F, Lai ML, Faa G, Mariotti S. The diagnostic value for differentiated thyroid carcinoma metastases of thyroglobulin ( $\mathrm{Tg}$ ) measurement in washout fluid from fine-needle aspiration biopsy of neck lymph nodes is maintained in the presence of circulating anti-Tg antibodies. J Clin Endocrinol Metab 2006;91:13641369 .

54. Duval M, Zanella AB, Cristo AP, Faccin CS, Graudenz MS, Maia AL. Impact of serum tsh and anti-thyroglobulin antibody levels on lymph node fine-needle aspiration thyroglobulin measurements in differentiated thyroid cancer patients. Eur Thyroid J 2017;6:292-297. 